\title{
ON Q-ALGEBRAS AND SPECTRAL ALGEBRAS
}

\author{
LOURDES PALACIOS, REYNA MARÍA PÉREZ-TISCAREÑO ${ }^{\dagger}$ AND CARLOS SIGNORET
}

\author{
Date of Receiving : : 06.04. 2016 \\ Date of Revision : $\quad$ 05. 05. 2016 \\ Date of Acceptance : 06.05 .2016
}

\begin{abstract}
T. Palmer gave the definition of a spectral seminorm and some equivalent statements for the seminormed algebras to be spectral. Some of these statements have been studied in the locally $m$-convex case and in the locally pseudoconvex case. In this paper we give some analogous results for the locally $m$-pseudoconvex algebras. Moreover, different proofs for some known results are given.
\end{abstract}

\section{Introduction}

In 1947 I. Kaplansky introduced in [9] the notion of Q-algebra (more exactly of $Q$-ring), that is a topological algebra $E$ in which the set $\operatorname{Qinv}(E)$ of quasi-invertible elements of $E$ is open. Examples of $Q$-algebras can be found in [5] and [6]. It is well known that every Banach algebra is also a $Q$-algebra. The importance of $Q$-algebras has been given, for example in [7], [8],[10]. Nowadays $Q$-algebras are involved in modern differential geometry (see [12, Chapter XI]) and play a significant role in PDE's theory, operator theory, and the $\Psi^{*}$-quantization (see $[7$, p. 73]).

In 1987 V.Mascioni [13] gave several equivalent conditions for a unital complex normed algebra to be a $Q$-algebra. Later on T. W. Palmer introduced in [14, p. 294] the notion of a spectral algebra as an algebra $E$ in which there exists a seminorm (spectral seminorm) $p$ such that $r(x) \leqslant p(x)$ for each $x \in E$, here $r(x)$ denotes the spectral radius of $x$. He gave there analogous results to V. Mascioni for seminormed algebras and M. Fragoulopoulou in [6, p. 85, Theorem 3.1] for locally $m$-convex algebras.

Several characterizations of locally $m$-pseudoconvex $Q$-algebras are given in the present paper.

2010 Mathematics Subject Classification. 46H05, 46H20.

Key words and phrases. Topological algebras, locally $m$-pseudoconvex algebras, spectral algebras, $Q$-algebras, spectral algebras.

Communicated by. Mati Abel

${ }^{\dagger}$ Corresponding author. 